\title{
Should patients with unresectable gallbladder cancer with hilar involvement undergo unilateral or bilateral percutaneous biliary drainage in the setting of cholangitis?
}

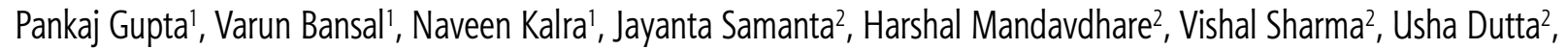 \\ Rakesh Kochhar², Manavjit Singh Sandhu' ${ }^{1}$
}

'Department of Radiodiagnosis and Imaging, Postgraduate Institute of Medical Education and Research (PGIMER), Chandigarh, India 2Department of Gastroenterology, Postgraduate Institute of Medical Education and Research (PGIMER), Chandigarh, India

\begin{abstract}
Aim of the study: This study aimed to evaluate the outcomes of patients with unresectable gallbladder cancer (GBC) with hilar involvement and cholangitis undergoing percutaneous transhepatic biliary drainage (PTBD).

Material and methods: This retrospective study comprised consecutive patients with unresectable GBC with cholangitis who underwent PTBD. The procedures were categorized as unilateral or bilateral. Bilateral PTBD was classified as simultaneous or sequential. The mean reduction in bilirubin at two weeks was recorded. Complications and mean overall survival were also recorded.

Results: Thirty-three patients (mean age 54.5 years, 12 males) were included. Thirty patients underwent unilateral drainage. Sequential drainage of the contralateral system was performed in 11 patients. Simultaneous bilateral PTBD was performed in 3 patients. PTBD was technically successful in all patients. Mean reduction in bilirubin was $41.5 \%$ in the unilateral group. The fall of bilirubin in the simultaneous bilateral PTBD group was $39 \%$. The mean follow-up duration was 36.5 days. No major complications were encountered. At the last follow-up, 7 patients were alive. The mean overall survival was 34.6 days.

Conclusions: Patients with unresectable GBC and cholangitis frequently require bilateral drainage. However, prospective studies should be performed to evaluate whether a sequential or simultaneous PTBD should be performed.
\end{abstract}

Key words: gallbladder cancer, biliary drainage, PTBD, cholangitis.

Address for correspondence:

Dr. Pankaj Gupta, Assis. Prof., GE Radiology, Department of Radiodiagnosis and Imaging, PGIMER, Chandigarh, India, phone: +91-1722756602, 7087003417, e-mail: Pankajgupta959@gmail.com

\section{Introduction}

Gallbladder carcinoma $(\mathrm{GBC})$ is a rare disease. However, in India, it is one of the most common gastrointestinal malignancies in females [1]. The condition commonly presents at an advanced stage and has an abysmal prognosis $[2,3]$. Biliary drainage represents an important palliation strategy for these patients [4]. In general, endoscopic drainage is the preferred choice for malignant lower end biliary obstruction [5]. In patients with GBC with perihilar involvement, percutaneous transhepatic biliary drainage (PTBD) is better than endoscopic drainage [6]. Patients with involvement of the primary confluence may undergo unilateral or bilateral PTBD [7-14]. Although data from patients with GBC are scarce, previous studies with a predominance of patients with cholangiocarcinoma had conflicting results regarding unilateral vs. bilateral drainage. A few series have shown that there is no benefit of bilateral drainage, while others suggested that bilateral drainage may be better [7-14]. A recent study that included a significant number of patients with GBC concluded that there was 
no difference in the quality of life or reduction of bilirubin in patients undergoing bilateral PTBD [11].

Cholangitis is a dreaded complication of unrelieved biliary obstruction. Timely biliary drainage is vital for the management of cholangitis $[15,16]$. However, there are no data to guide whether patients with GBC who have isolation of the right and left ductal system by hilar involvement should undergo unilateral or bilateral drainage for the management of cholangitis. We conducted this audit to analyze the data regarding the relative utilization of unilateral and bilateral PTBD in patients with unresectable GBC with hilar involvement presenting with cholangitis.

\section{Material and methods}

The local ethics committee approved the study. A retrospective review of data of consecutive patients who underwent PTBD in an interventional radiology unit of a tertiary care referral hospital between May 2018 and March 2020 was performed. Patients who underwent PTBD for unresectable GBC with perihilar involvement and cholangitis were included. Patients with resectable disease, patent primary confluence, and endoscopic drainage before percutaneous drainage were excluded from the study. Also, patients with involvement of bilateral secondary confluences were excluded.

The diagnosis of GBC was based on imaging evaluation and fine-needle aspiration from the GB mass. As per the institutional policy, all patients with unresectable GB lesions undergo cytological diagnosis to offer them palliative treatment or neoadjuvant chemotherapy. The diagnosis and grading of cholangitis were performed according to the Tokyo guidelines 2018 [15].

PTBD was done according to the protocol described previously [16]. All the patients planned for PTBD were admitted and given pre-procedure intravenous antibiotics and hydration. PTBD was done under combined ultrasound and fluoroscopic guidance. A detailed evaluation of imaging was done to select the appropriate duct. In patients undergoing a unilateral procedure in the first session, left-sided PTBD was preferred. This preference is based on the unit's experience as well as a recent publication suggesting increased pain and poor quality of life in patients undergoing right PTBD [17]. However, patients who had cholangitis or evidence of unilateral biliary sepsis (in the form of echogenic debris/adjacent small cholangitis abscesses) underwent PTBD of that side.

\section{PTBD procedure}

After cleaning the local site with a $10 \%$ betadine solution and draping the area, $10 \mathrm{ml}$ of $2 \%$ injection lignocaine was used for local anesthesia. The appropriate duct was accessed using an $18 \mathrm{G}$ needle under realtime ultrasound guidance. After allowing some bile to drain passively, minimal diluted contrast $(2-3 \mathrm{ml})$ was injected under fluoroscopic guidance. If pus came out, no contrast was injected. A hydrophilic guidewire ( 0.035 inches, Terumo) was inserted through the needle into the ductal system until the site of the block. The needle was removed, keeping the wire in the ductal system. After dilatation of the skin tract with a $6 \mathrm{~F}$ fascial dilator, a $5 \mathrm{~F}$ angiographic catheter (multipurpose catheter, Cook) was inserted over the guidewire. Following this, a 0.035 -inch stiff guidewire (Amplatz, Cook) was placed through the catheter into the ductal system. The skin-subcutaneous tract was dilated sequentially with $7 \mathrm{~F}$ and $8 \mathrm{~F}$ dilators. An $8 \mathrm{~F}$ external biliary catheter was finally inserted, and the position was confirmed with contrast injection under fluoroscopy. The catheter was fixed using sutures and left to external drainage (Fig. 1). Internalization was performed in a separate session 3-7 days apart, in afebrile patients. Patients requiring subsequent contralateral PTBD underwent interval drainage using the same protocol (Fig. 2).

Patients were observed for complications (hemobilia, pericatheter leakage, fever, and worsening of cholangitis). The following details were recorded: 1) extent of involvement of the ductal system (involvement of primary vs. secondary confluence), 2) severity of cholangitis, 3) laterality of drainage - left vs. right vs. bilateral [simultaneous (performed in the same session) vs. sequential (performed in two separate sessions)], 4) those requiring contralateral PTBD - the interval between the two procedures was also recorded. The post-procedure bilirubin levels at two weeks were recorded. Baseline and post-procedure blood cultures were evaluated. Mean follow-up and outcomes of patients were recorded.

\section{Results}

During the study period, PTBD was performed in 168 patients. Fifty-seven patients underwent PTBD for GBC. Out of these, 33 patients with unresectable GBC, who underwent PTBD for hilar involvement in the setting of cholangitis, were included.

The mean age was 54.5 years (range, 30 to 85 years). There were 12 male and 21 females. Mean baseline total leucocyte count was 12,281 cells $/ \mathrm{mm}^{3}$ (range, 360030,100 cells $/ \mathrm{mm}^{3}$ ) and bilirubin was $11.7 \mathrm{mg} / \mathrm{dl}$ (range, $0.8-31.5 \mathrm{mg} / \mathrm{dl}$ ). Blood culture results at baseline were available in 22 patients. The baseline blood cultures 

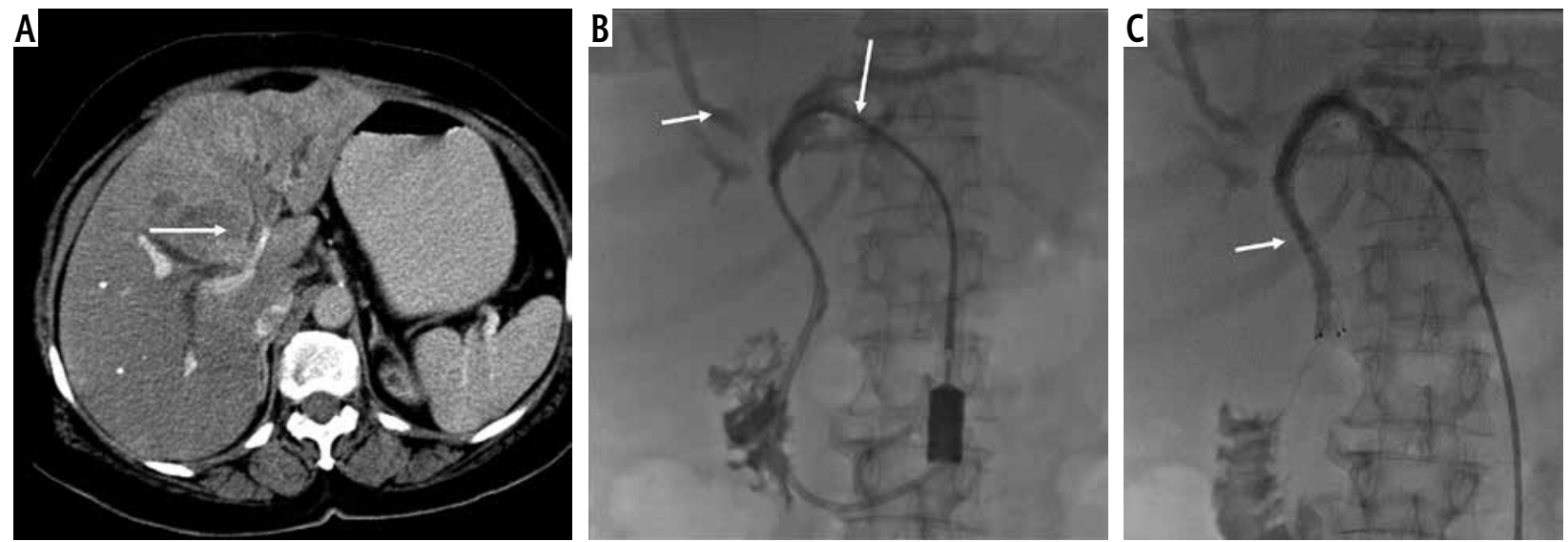

Fig. 1. Unilateral PTBD. A) Axial CT image shows a mass causing block at the level of primary confluence (arrow). B) Left sided PTBD was done (arrow). The right system is undrained (short arrow). C) Later this patient recovered from cholangitis and underwent self-expanding metallic stent placement (arrow)
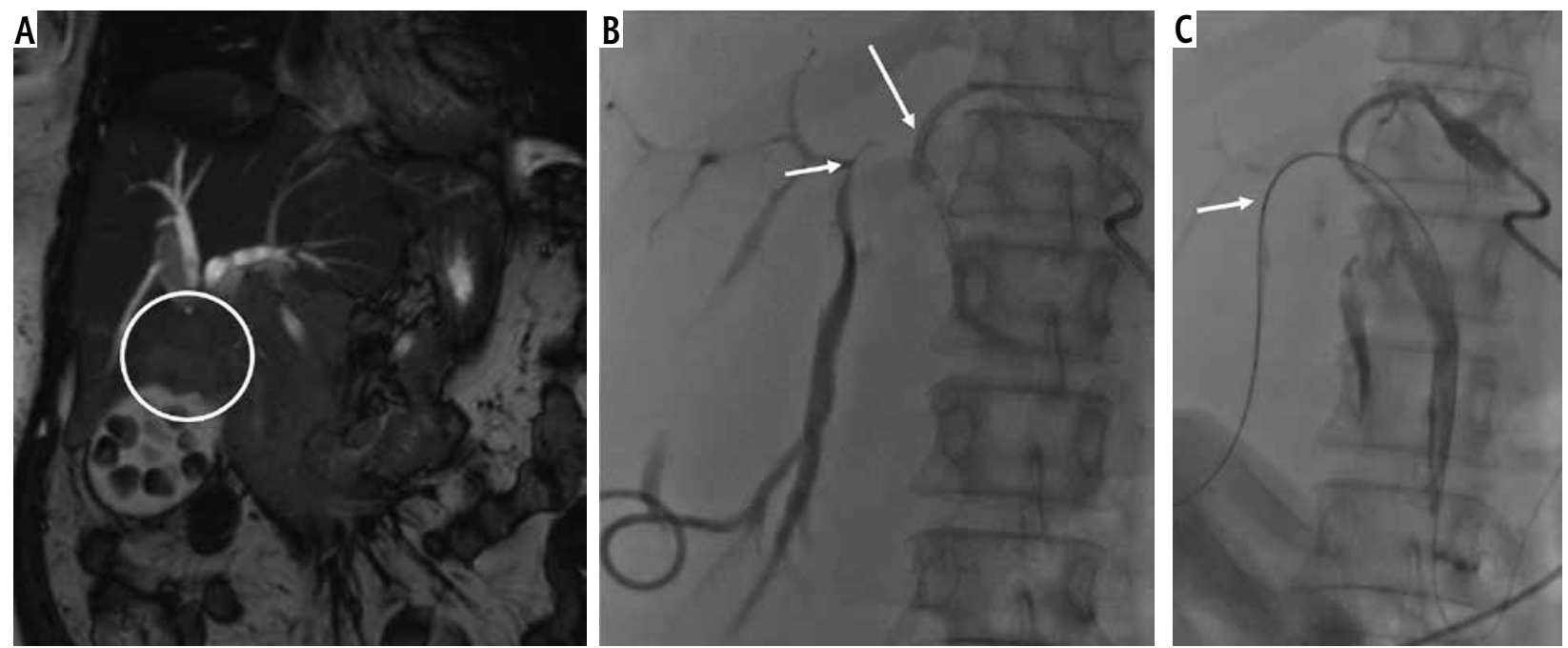

Fig. 2. Sequential bilateral PTBD. A) Coronal TRUFI MR image shows a mass arising from the neck of the gallbladder (circle) causing separation of right and left ductal systems. B) The patient had a left PTBD catheter in situ (done 1 week before, arrow). There was non-resolving moderate cholangitis. The right ductal system was accessed and opacified (short arrow). C) At a later date, the catheter was internalized. A wire was negotiated across the stricture into the duodenum (arrow)

were sterile in 15 patients. Metastatic disease was present in 20 patients at baseline.

Only the primary confluence was involved in $27 \mathrm{pa}-$ tients. The left secondary confluence was involved in 2 patients, and the right secondary confluence was involved in 4 patients. The indications for initial PTBD were mild cholangitis not responding to antibiotics in 23 patients, moderate cholangitis in 1 patient, and severe cholangitis in 9 patients.

The procedure was technically successful in all patients. Initial unilateral PTBD was performed in $30 \mathrm{pa}-$ tients. Three patients underwent simultaneous right and left PTBD. Of the 30 patients undergoing unilateral drainage in the $1^{\text {st }}$ session, 11 patients underwent subsequent drainage of the contralateral ductal system. Thus, in the overall group, $42.4 \%$ of the patients underwent bilateral drainage. The mean interval between the two sessions was 14.2 days (range, 3-50). Internalization was performed in 9 patients, and metallic stenting was done unilaterally in 2 patients.

Complications of PTBD were mild hemobilia in 3 patients, catheter slippage in 6 patients, and pericatheter leakage in 1 patient. There was no difference in the complications between the unilateral and bilateral groups.

Follow-up bilirubin at two weeks was available in 22 patients. Except for a patient with left-sided PTBD with severe cholangitis (baseline bilirubin of $2.7 \mathrm{mg} / \mathrm{dl}$ ) who had an increase in bilirubin $(4.3 \mathrm{mg} / \mathrm{dl}$, an increase of 59.2\%), all the patients had a fall in bilirubin. There was a mean reduction of bilirubin by $41.5 \%$ (range, $3.4-92.5 \%)$ in the unilateral group. In three patients who underwent bilateral PTBD in the $1^{\text {st }}$ session, follow-up bilirubin levels were available in 
Table 1. Baseline characteristics, procedure details and clinical outcomes of patients

\begin{tabular}{|c|c|}
\hline Characteristics & Number \\
\hline Age (years), mean (range) & $54.5(30-85)$ \\
\hline Males : females & $12: 21$ \\
\hline \multicolumn{2}{|l|}{ Grade of cholangitis } \\
\hline Mild & 23 \\
\hline Moderate & 1 \\
\hline Severe & 9 \\
\hline \multicolumn{2}{|l|}{ Level of obstruction } \\
\hline Primary confluence & 27 \\
\hline Left secondary & 2 \\
\hline Right secondary & 4 \\
\hline \multicolumn{2}{|l|}{ PTBD site } \\
\hline Unilateral & 30 \\
\hline Right & 16 \\
\hline Left & 14 \\
\hline \multicolumn{2}{|l|}{ Bilateral } \\
\hline Simultaneous & 3 \\
\hline Sequential & 11 \\
\hline Mean $\%$ reduction of bilirubin & $41.5 \%$ \\
\hline \multicolumn{2}{|l|}{ Baseline blood culture } \\
\hline Sterile & 15 \\
\hline Positive & 7 \\
\hline Post-PTBD blood culture positivity & $5^{*}$ \\
\hline \multicolumn{2}{|l|}{ Complication } \\
\hline Hemobilia & 3 \\
\hline Pericatheter leakage & 1 \\
\hline Catheter slippage & 6 \\
\hline Mean follow-up (days) & 36.5 \\
\hline Overall survival & 7 \\
\hline Mean overall survival (days) & 34.6 \\
\hline
\end{tabular}

two patients. The fall of bilirubin in these patients was $38.9 \%$ (baseline bilirubin, $18 \mathrm{mg} / \mathrm{dl}$ ) and $39.9 \%$ (baseline bilirubin, $2.8 \mathrm{mg} / \mathrm{dl}$ ). The data regarding both pre- and post-procedure blood culture were available in 17 patients. Following PTBD, blood culture yielded organisms in 5 patients. However, none of the patients had clinical worsening of cholangitis. Five patients were lost to follow-up. The mean follow-up duration was 36.5 days (range, 0-226). At the time of the last follow-up, seven patients were alive. The mean overall survival was 34.6 days. Table 1 shows the main characteristics of the patients.

\section{Discussion}

This is one of the few studies evaluating the role of PTBD in patients with GBC. We evaluated outcomes of PTBD in GBC patients with cholangitis who had isolation of the right and left ductal system. We mainly focused on the utilization of unilateral vs. bilateral PTBD. We found that $42.4 \%$ of the patients required bilateral drainage.

However, only 9.1\% (3/33) patients underwent bilateral drainage in the $1^{\text {st }}$ session. In the rest of the patients, contralateral PTBD was done when the patient failed to improve clinically with unilateral drainage. The interval between the two procedures was 14.2 days (range, 3-50). The overall outcome in both the groups was poor, with a mean survival of 34.6 days.

There is a lack of studies evaluating the incidence of cholangitis in GBC. Most of the studies report the incidence of post-intervention cholangitis in the setting of malignant biliary obstruction. In the study by Saluja et al., $43 \%$ of the GBC patients had cholangitis at baseline evaluation, requiring biliary drainage [5]. The grade of cholangitis was not reported. In our study, most patients had mild cholangitis at baseline evaluation. They were referred for biliary drainage as they did not improve with medical treatment. Ten (30.3\%) patients had moderate and severe cholangitis requiring early/urgent biliary drainage. In many patients, mild cholangitis requiring biliary drainage may be secondary to the poor general condition of these patients, and these patients may progress to moderate and severe cholangitis [18].

In a meta-analysis of nine studies [including two randomized controlled trials (RCTs)], bilateral stenting was associated with a significantly reduced re-intervention rate. Other endpoints, including technical success, early and late complications, and stent dysfunction, were not significantly different between the two groups [19]. The two RCTs that evaluated the efficacy of unilateral vs. bilateral endoscopic drainage had conflicting results. An earlier trial by De Palma et al. including 157 patients suggested that bilateral stenting $(n=78)$ had a lower technical success rate and a significantly higher rate of early cholangitis. There was no significant difference in survival between the two groups [12]. In this study, there were 15 (19\%) patients in the unilateral group and $16(20.5 \%)$ patients in the bilateral group with GBC. In the study by Lee et al., out of the 133 patients, 66 underwent unilateral metallic stenting, and 67 underwent bilateral metallic stenting [14]. The authors found that patients who underwent bilateral drainage required fewer reinterventions. This study included $12(17.9 \%)$ and $12(18.2 \%)$ patients with GBC in the unilateral and bilateral groups, respectively. 
In a non-randomized study on patients with malignant hilar strictures (Bismuth-Corlette type II), outcomes of 39 patients undergoing treatment with unilateral or bilateral endoscopic drainage were evaluated [20]. There were 32 patients with cholangiocarcinoma and seven patients with GBC. There was a significantly greater mean reduction in bilirubin at one week following the procedure in patients with bilateral plastic stenting $(n=18)$. There was no significant difference in the early complications and other clinical outcomes. In another study comparing the outcomes of patients with malignant hilar strictures undergoing unilateral or bilateral metallic stenting, 82 patients were included [8]. Sixty-five patients underwent unilateral drainage and 17 patients underwent bilateral drainage. There were $11(16.9 \%)$ and $5(29.4 \%)$ patients with GBC in the unilateral and bilateral group, respectively. The incidence of post-procedure cholangitis with liver abscesses was significantly higher in the bilateral group. There was no significant difference in other complications. However, none of the published studies reported the subgroup analysis for patients with GBC. Additionally, the subgroup analysis of patients with baseline cholangitis has not been reported in the published studies.

In a study by Gamanagatti et al. on 49 patients with malignant biliary obstruction who underwent percutaneous biliary drainage, the majority $(35,71.4 \%)$ had GBC [11]. Technical success was achieved in all patients. There was no significant difference in the clinical success or quality of life between patients undergoing unilateral or bilateral drainage. The presence of cholangitis as an indication of biliary drainage was not explicitly reported. The survival in our group (mean, 34.5 days) of patients was lower than that reported in the previous series of patients with GBC by Saluja et al. (median, 60 days) and Gamanagatti et al. (median, 90 days) $[6,11]$. The lower mortality in the previous studies could be explained by the fact that patients presenting with cholangitis were not specifically studied. Additionally, in the study by Gamanagatti et al., the causes of malignant biliary obstruction other than GBC were also included [11]. It is well known that GBC has a worse prognosis compared with other causes of malignant biliary obstruction [21]. In our group, $27.3 \%$ of patients had severe cholangitis. These patients have a uniformly poor outcome. Additionally, more than $50 \%$ of the patients had metastatic disease at baseline.

There were several limitations to our study. The sample size was small. However, considering the rarity of GBC in most countries and the fact that we included a specific subgroup of GBC, this may not undermine the importance of this study. As only three patients underwent simultaneous bilateral PTBD, bilirubin reduction as well as overall survival could not be compared with the unilateral group. The decision to perform PTBD of the contralateral system was not based on a definite threshold, limiting the interpretation of these results. However, understandably, the comparison of outcomes in this type of patient cohort are best suited for an RCT. Quality of life assessment was not performed as a part of this study.

In conclusion, our study suggests that a significant proportion of patients with GBC and hilar involvement may require upfront bilateral PTBD in the setting of cholangitis. However, considering several limitations, the results must be viewed with caution and should ideally be confirmed by a prospective study or an RCT.

\section{Disclosure}

The authors declare no conflict of interest.

\section{References}

1. Dutta U, Bush N, Kalsi D, et al. Epidemiology of gallbladder cancer in India. Chinese Clin Oncol 2019; 8: 33.

2. Ertel AE, Bentrem D, Abbott DE. Gall bladder cancer. Cancer Treat Res 2016; 168: 101-120.

3. Gupta P, Kumar M, Sharma V, et al. Evaluation of gallbladder wall thickening: a multimodality imaging approach. Exp Rev Gastroenterol Hepatol 2020; 14: 463-473.

4. Gupta P, Gupta J, Kumar MP. Imaging of obstructive jaundice: what a radiologist needs to know before doing a percutaneous transhepatic biliary drainage. J Clin Interv Radiol ISVIR 2020; 4: 31-37.

5. Ba Y, Yue P, Leung JW, et al. Percutaneous transhepatic biliary drainage may be the preferred preoperative drainage method in hilar cholangiocarcinoma. Endosc Int Open 2020; 8: E203-210.

6. Saluja SS, Gulati M, Garg PK, et al. Endoscopic or percutaneous biliary drainage for gallbladder cancer: a randomized trial and quality of life assessment. Clin Gastroenterol Hepatol 2008; 6: 944-950.

7. Naitoh I, Ohara H, Nakazawa T, et al. Unilateral versus bilateral endoscopic metal stenting for malignant hilar biliary obstruction. J Gastroenterol Hepatol 2009; 24: 552-557.

8. Iwano H, Ryozawa S, Ishigaki $\mathrm{N}$, et al. Unilateral versus bilateral drainage using self-expandable metallic stent for unresectable hilar biliary obstruction. Dig Endosc 2011; 23: 43-48.

9. Inal M, Akgül E, Aksungur E, Seydaoglu G. Percutaneous placement of biliary metallic stents in patients with malignant hilar obstruction: unilobar versus bilobar drainage. J Vasc Interv Radiol 2003; 14: 1409-1416.

10. Gwon DI, Ko GY, Sung KB, et al. Percutaneous biliary metallic stent placement in patients with unilobar portal vein occlusion caused by advanced hilar malignancy: outcome of unilateral versus bilateral stenting. AJR Am J Roentgenol 2011; 197: 795801.

11. Gamanagatti S, Singh T, Sharma R, et al. Unilobar versus bilobar biliary drainage: Effect on quality of life and bilirubin level reduction. Indian J Palliat Care 2016; 22: 50-62. 
12. Chang WH, Kortan P, Haber GB. Outcome in patients with bifurcation tumors who undergo unilateral versus bilateral hepatic duct drainage. Gastrointest Endosc 1998; 47: 354-362.

13. De Palma GD, Galloro G, Siciliano S, et al. Unilateral versus bilateral endoscopic hepatic ducts drainage in patients with malignant hilar biliary obstrcution: results of a prospective, randomized, and controlled study. Gastrointest Endosc 2001; 53: 547-553.

14. Lee TH, Kim TH, Moon JH, et al. Bilateral versus unilateral placement of metal stents for inoperable high grade malignant hilar biliary strictures: a multicenter, prospective, randomized study (with video). Gastrointest Endosc 2017; 86: 817-827.

15. Miura F, Okamoto K, Takada T, et al. Tokyo Guidelines 2018: initial management of acute biliary infection and flowchart for acute cholangitis. J Hepatobiliary Pancreat Sci 2018; 25: 31-40.

16. Gupta P, Maralakunte M, Rathee S, et al. Percutaneous transhepatic biliary drainage in patients at higher risk for adverse events: experience from a tertiary care referral center. Abdom Radiol (NY) 2020; 45: 2547-2553.

17. Castiglione D, Gozzo C, Mammino L, et al. Health-related quality of life evaluation in "left" versus "right" access for percutaneous transhepatic biliary drainage using EORTC QLQBIL-21 questionnaire: a randomized controlled trial. Abdom Radiol (NY) 2020; 45: 1162-1173

18. Ahmed M. Acute cholangitis - an update. World J Gastrointest Pathophysiol 2018; 9: 1-7.

19. Ashat M, Arora S, Klair JS, et al. Bilateral vs unilateral placement of metal stents for inoperable high-grade hilar biliary strictures: A systemic review and meta-analysis. World J Gastroenterol 2019; 25: 5210-5219.

20. Bulajic M, Panic N, Radunovic M, et al. Clinical outcome in patients with hilar malignant strictures type II Bismuth-Corlette treated by minimally invasive unilateral versus bilateral endoscopic biliary drainage. Hepatobiliary Pancreat Dis Int 2012; 11: 209-214.

21. Hundal R, Shaffer EA. Gallbladder cancer: epidemiology and outcome. Clin Epidemiol 2014; 6: 99-109. 